\title{
La danza como estrategia interdisciplinar de aprendizaje en los estudiantes de 5 a 7 años del colegio Holanda, sede Los Cacaos, Piedecuesta, Santander, Colombia
}

\section{The dance as a interdisciplinary learning strategy in the kids from Holanda School, Headquarters, Los Cacaos, Piedecuesta, Santander, Colombia}

\author{
Laura Lissette Duarte Ustman \\ Maestría en Educación \\ Colegio Holanda, Piedecuesta, Santander, Colombia \\ Correo electrónico: laduarte02@gmail.com
}

Recibido: 12 de octubre de 2017. Aceptado: 5 de diciembre de 2017

\section{Cómo citar este artículo}

Duarte, L.L. (2018). La danza como estrategia interdisciplinar de aprendizaje en los estudiantes de 5 a 7 años del colegio Holanda, sede Los Cacaos, Piedecuesta, Santander, Colombia. Espiral, Revista de Docencia e Investigación, 8(1) 65 - 79

\section{Resumen}

Tipología textual y objetivo. El presente artículo de investigación es el resultado de un estudio de tipo cualitativo, desde un enfoque etnográfico. Tiene como objetivo caracterizar la efectividad de la danza como estrategia interdisciplinar.

Metodología. Se aplicó el enfoque etnográfico a procesos educativos relacionados con la práctica docente a niños del aula multigrado de preescolar, primero y segundo, bajo el modelo llamado Escuela Nueva, implementado en el Colegio Holanda, sede Los Cacaos del municipio de Piedecuesta, Santander, Colombia. Se empleó como instrumentos las entrevistas, la observación de clase, así como momentos pedagógicos que resaltan algunas de las danzas representativas del folclore colombiano como estrategia interdisciplinar. Se evidenció la intervención de esta estrategia aplicada a los estudiantes con la participación de los padres de familia y directivos.

Resultados. Este trabajo se desarrolló en un período de dos años; su aplicación permitió generar espacios de expresión y un aprendizaje significativo. Se logró potenciar momentos de atención y participación de los estudiantes, además del descubrimiento de nuevas estrategias para la enseñanza y aprendizaje relacionadas con una de las bellas artes.

Palabras clave: Danza, estrategia interdisciplinar, didáctica, folclore colombiano.

\section{Abstract}

Textual typology and objective. The present research article is the result of a qualitative study, from an ethnographic approach. Its objective is to characterize the effectiveness of dance as an interdisciplinary strategy.

Methodology. The ethnographic approach was applied to educational processes related to teaching practice to children in the multigrade pre-school classroom, first and second, under the model called "Escuela Nueva (New School)", implemented in Colegio Holanda, sede Los Cacaos in the municipality of Piedecuesta, Santander, Colombia. Interviews and class observation were used as instruments, as well as pedagogical moments that highlight some of the representative dances of Colombian folklore as an interdisciplinary strategy. The intervention of this strategy applied to students was evidenced with the participation of parents and managers.

Results. This work was developed over a period of two years; its application allowed the generation of spaces of expression and significant learning. It managed to enhance moments of attention and student participation, as well as the discovery of new strategies for teaching and learning related to one of the fine arts.

Keywords: Dance, interdisciplinary strategy, didactic, Colombian folklore. 


\section{Introducción}

Un aprendizaje despierta expectativa, curiosidad, atrae más que seguir una rutina monótona. La educación desde la danza permite sensibilizar de manera potencial a los niños de cualquier edad; construye espacios afables de conocimiento, de atención y de aprendizaje. Por tal razón es necesario fundamentar y desarrollar un estudio acerca de esta bella arte como estrategia interdisciplinar de enseñanza y aprendizaje.

Un punto de partido para la investigación, lo constituye este interrogante: ¿La danza es una estrategia interdisciplinar capaz de proyectar la enseñanza y el aprendizaje de los niños y niñas de 5 a 7 años del colegio Agroecológico Holanda, sede Los Cacaos? Entendimos que esta inquietud investigativa necesitaba una guía, un norte, por ello surgieron los objetivos. Uno inicial, el objetivo general: Determinar la efectividad de la danza como estrategia interdisciplinar en los niños y niñas de 5 a 7 años en la institución Colegio Agroecológico Holanda - sede Los Cacaos.

En concordancia, los objetivos que se pretendieron alcanzar fueron: Diagnosticar las concepciones y prácticas que se tienen en la escuela con relación a la danza como estrategia interdisciplinar de enseñanza - aprendizaje, además de relacionar la danza como proceso de formación didáctica con la proyección de la dimensión psicosocial. Y un segundo objetivo: Determinar los espacios y momentos pedagógicos producidos en interdisciplinariedad a partir de la danza.

La danza como estrategia interdisciplinar de enseñanza y aprendizaje permite como disciplina artística que transversaliza diferentes áreas de conocimiento, incrementar en los niños mejoras en aspectos como la expresión, dominio corporal y estético. Fue posible, en consecuencia, encontrar nuevos métodos de estudio en los que la relación de los conocimientos adqui- ridos ayudaron al estudiante a aprender y también a desaprender logrando enriquecer conocimientos y emociones. Veamos cómo sucedió este proceso:

\section{Metodología}

Los estudios cualitativos aportan información sobre las motivaciones profundas de las personas, cuáles son sus pensamientos y sus sentimientos; proporcionan información para adecuar el diseño metodológico de un estudio.

Bien sabido es que en procesos de las Ciencia Sociales y en educación es una de las metodologías más usadas en los ámbitos investigativos. En este sentido, todo cambio o reforma que se desarrolla en las escuelas debe basarse en investigaciones a fin de establecer el qué, el cómo y el hacia dónde. De esta forma orientan la toma de decisiones, y permite a los docentes construir y reconstruir los procesos necesarios para fortalecer la cultura desde la escuela.

Por lo anterior, el desarrollo de este estudio se hizo partiendo de la investigación cualitativa, orientada desde un enfoque etnográfico, aplicada a unos procesos educativos donde los niños son los agentes del proceso de investigación.

El trabajo pues, se realizó con un grupo de niños y niñas en un contexto de aula modelo Escuela Nueva que pertenecen al grado preescolar, primero y segundo, bajo la metodología multigrado. Se usó como instrumentos de información la observación y las entrevistas a diversos públicos (docentes - padres de familia y directivos). Lo anterior porque es necesario tener en cuenta que si los investigadores no miran hacia la escuela, puede perder no solo un área de aplicación de conocimientos producto del quehacer investigativo, sino un espacio a partir del cual se postulen teorías pedagógicas. De ahí la importancia de pensar la danza como estrategia, para determinar su eficacia en el proceso de aprendizaje de otras disciplinas distintas a la artística, porque de acuerdo a Woods (1987, p. 34) 
La etnografía infiere entre la investigación educativa y la práctica docente, descripción del modo de vida de un grupo. Se interesa por lo que la gente hace, cómo se comporta, cómo interactúa. Se propone descubrir sus creencias, valores, perspectivas, motivaciones y como se desarrolla (p. 18).

De otra parte, Ogando (2004, p. 1) cita a Rinaudo, quien afirma que si maestros y profesores no atienden a las producciones de la investigación, limitan considerablemente el marco de conocimientos que informa sus decisiones instructivas y con ello disminuyen también la calidad de sus intervenciones pedagógicas.

\section{Ezpeleta (2004) por su parte agrega:}

Las innovaciones constituyen la manera a través de la cual los modelos de reforma se hacen presentes en las escuelas. Las orientaciones reformistas sancionadas en los años noventa encuentran en las mismas el medio para inducir las transformaciones que consideran deseables (p. 404).
En este sentido Ezpeleta respalda nuestra propuesta en la medida que plantea el concepto y circunstancia de la innovación como la esencia de la transformación del sistema educativo convencional.

Por décadas los estudiosos del cambio educativo coinciden en señalar que la innovación en las prácticas docentes tiene mayor posibilidad de éxito y arraigo cuando surge de las propias escuelas. Advierten también que la particular confluencia de factores que la origina, limitan sus posibilidades de ampliación o generalización. Por el contrario, Huberman, Fullan y Stiegelbauer, citados en Ogando (2004), afirman que cuando el cambio es inducido y se propone afectar al conjunto de un nivel-como es el caso de una reforma-, hay una tendencia a la resistencia por parte de los actores y de las estructuras escolares (p. 11).

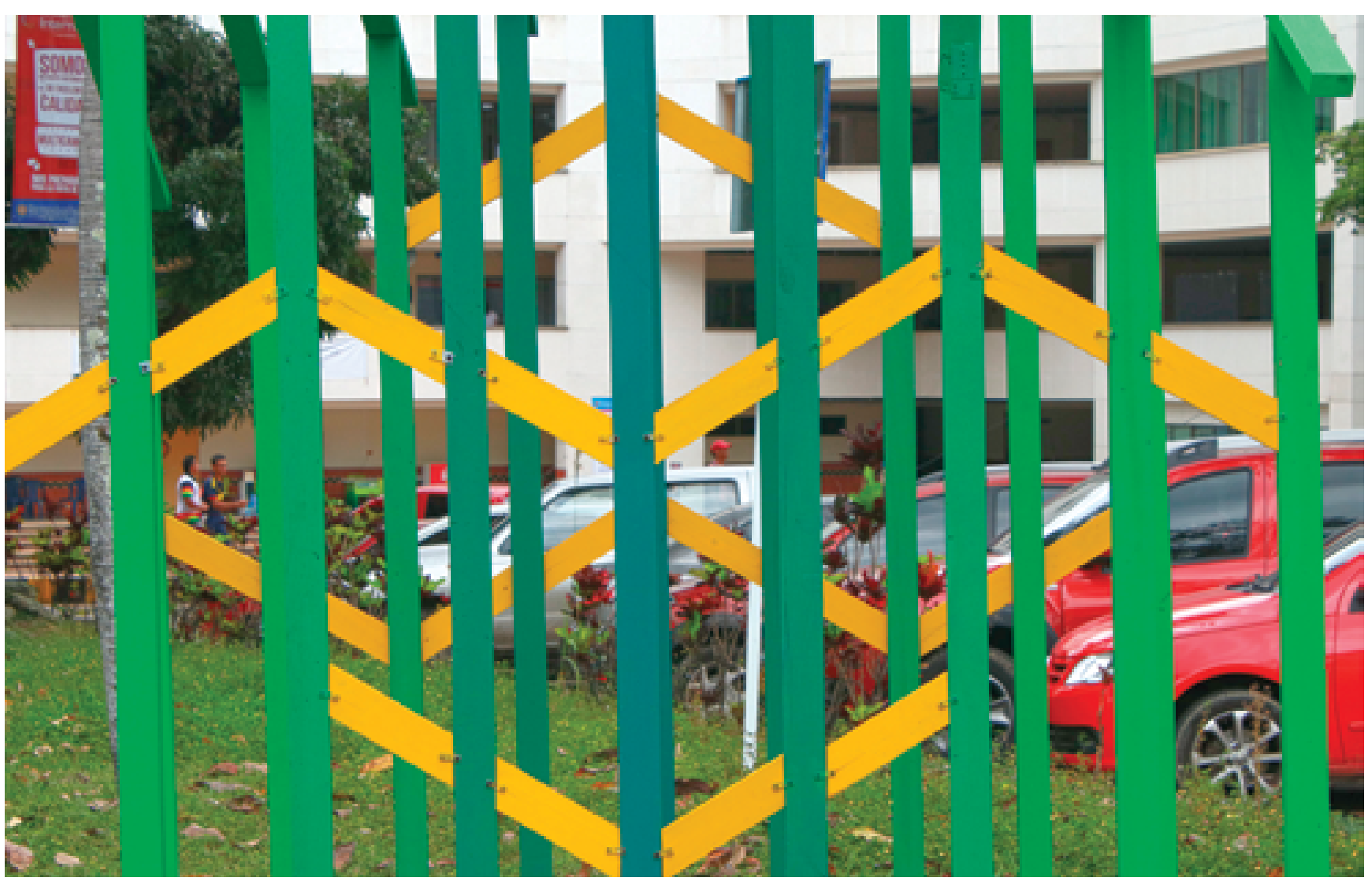

Proyecto Mamba Verde. 
De otra parte, Woods (1987) deduce: "entre la etnografía y la enseñanza hay ciertos paralelismos adaptables entre sí. Son una mezcla entre arte y ciencia. Ambas conciernen en contar una historia, ambas investigan, analizan y organizan" (p, 20). En estos procesos de transformación se vio cómo los profesores innovaban de manera individual, resultado de un proceso permisivo más que participativo. Frente a esta cuestión, docentes e investigadores toman conciencia de la importancia del centro docente para poner en marcha procesos de cambio realmente exitoso. Por eso la experiencia investigativa que se presenta, parte desde el aula y de una concepción diferente de la didáctica, más universal y transversal para determinar cómo a través de la danza se generan aprendizajes de otras disciplinas o áreas académicas.

\section{Proceso de la investigación}

Siguiendo a Flores y Gómez (1999), el presente estudio se consolida en las fases que muestra la figura 1.

Figura 1. Secuencia investigativa.

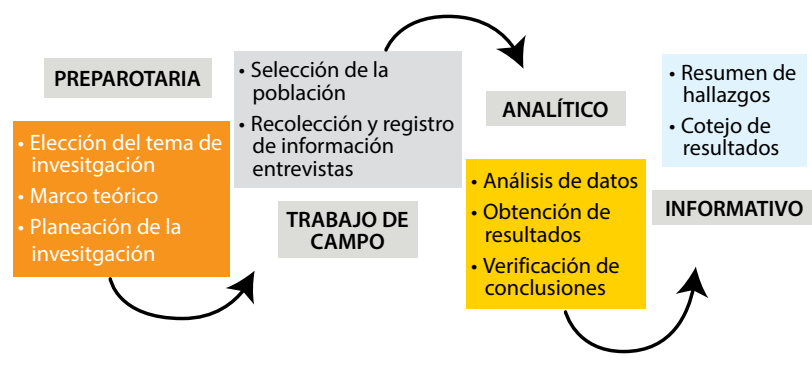

Fuente: Tomado de Flores y Gómez (1999)

\section{Población, participantes y selección de la muestra}

El colegio Agroecológico Holanda, en la vereda Holanda del municipio de Piedecuesta del departamento de Santander, Colombia, es una institución oficial de carácter mixto con énfasis en agroecología y cuenta con siete sedes: Holanda, San Miguel, La Esperanza, Los Cacaos, El Duende, Mesitas de San Javier y La Navarra.
La comunidad que forma parte de la sede Los Cacaos se dedica principalmente a la avicultura, ganadería y agricultura. Los niños en su mayoría pertenecen a varios estratos, es decir, se encuentran niños de zona rural estratos (01-2), niños campesinos, niños con abandono de hogar, niños desplazados por la violencia en Colombia y Venezuela, y niños de zona urbana (3 - 6) que por cambio de domicilio, departamento o país llegan a la escuela.

En la actualidad la sede cuenta con 55 estudiantes de preescolar a quinto, dos salones y dos docentes y la auxiliar de servicio del restaurante escolar. El horario de estudio es establecido para todos los estudiantes de lunes a viernes de 7:00 a.m. a 3:00 p.m. siendo el primer colegio con jornada continua en el municipio de Piedecuesta hasta el momento.

La muestra de los estudiantes se elige por conveniencia y está representada en 18 niños de los grados preescolar, primero y segundo de básica primaria, que son los estudiantes con quienes se ejerce la labor docente, que representan el $100 \%$ del total de la población. También se tuvo en cuenta a los padres de familia de los grupos que forman la población, seleccionados de manera aleatoria, representando el $60 \%$ de los padres.

La población de la presente investigación estuvo compuesta por 18 estudiantes que son del grado preescolar, primero y segundo. Empleando la herramienta Excel histograma, se representaron los grupos que participaron en el aula multigrado para el estudio de investigación y se especificaron niños y niñas que están cursaron los grados de preescolar, primero y segundo. 

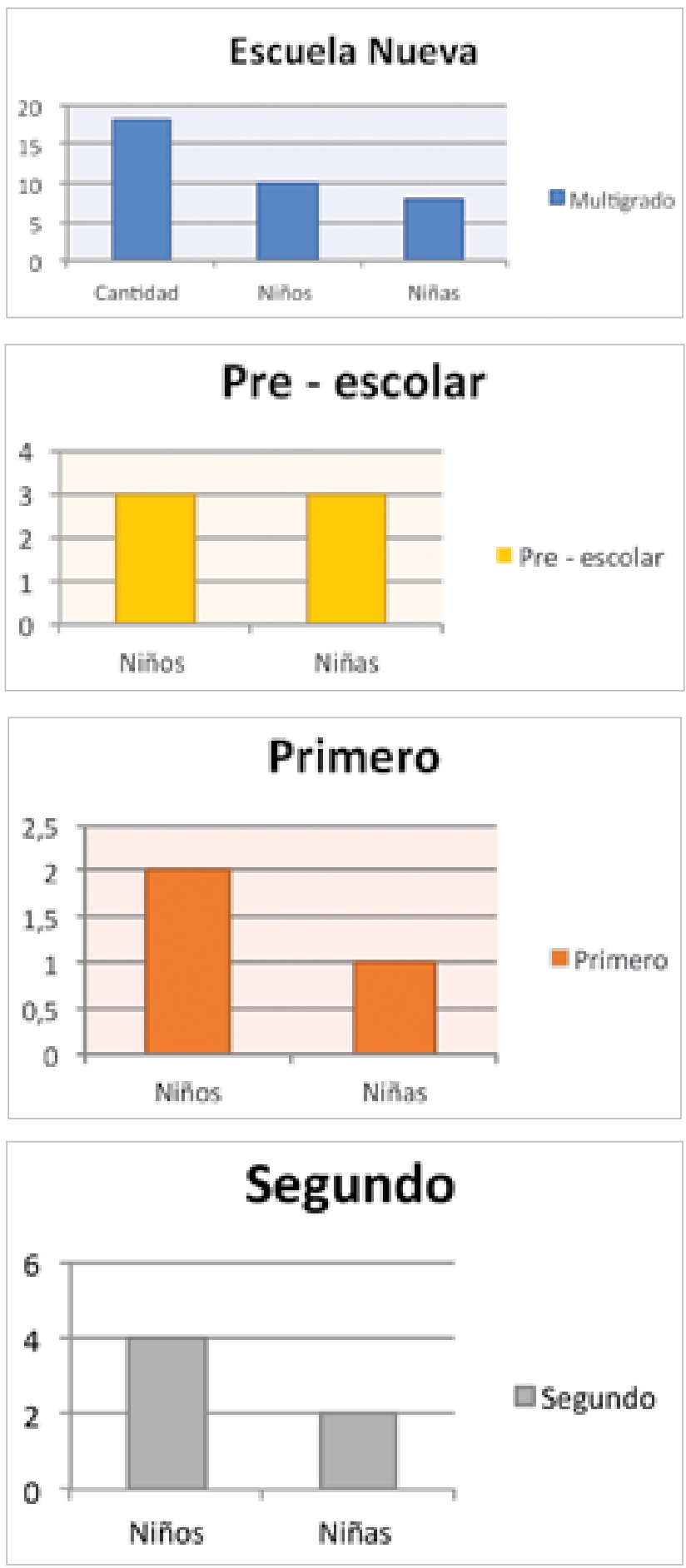

Fuente: Elaboración propia.

\section{Instrumentos para la recolección de la información}

Las técnicas de la investigación se seleccionaron por un conjunto de factores adicionales, entre los cuales incidió la naturaleza de la pre- gunta formulada. Además, intervinieron otros factores que deben ser considerados: el tiempo disponible, los recursos y quién los otorga, el conocimiento previo acumulado sobre el tema específico y el grado de encadenamiento del estudio concreto con otros.

Las técnicas cualitativas, en consecuencia, nos proporcionaron una mayor profundidad en la respuesta y así una mayor compresión del fenómeno estudiado. Estas técnicas normalmente suponen un menor costo que las técnicas cuantitativas, son de más rápida ejecución, permiten más flexibilidad en su aplicación y favorecen establecer un vínculo más directo con los sujetos.

En esta investigación se utilizaron los siguientes instrumentos

\begin{tabular}{ll}
\hline \multicolumn{1}{|c}{ Instrumentos } & \multicolumn{1}{c}{ Aplicados a } \\
$\begin{array}{l}\text { Ficha diagnóstica } \\
\text { del estudiante }\end{array}$ & $\begin{array}{l}\text { Caracterización de los acto- } \\
\text { res del proceso educativo } \\
\text { y percepciones de los estu- } \\
\text { diantes en relación con las } \\
\text { motivaciones para ir al aula. }\end{array}$ \\
& Diario pedagógico docente \\
\hline Entrevista & Padres de familia \\
\hline $\begin{array}{l}\text { Observación de } \\
\text { clase }\end{array}$ & $\begin{array}{l}\text { Directivos - Rector / Coordi- } \\
\text { nadora } \\
\text { realiza la investigación }\end{array}$ \\
\hline
\end{tabular}

\section{La observación participante}

Campoy y Gomes (2009) la conceptualizan como "aquella en la que el observador participa de manera activa dentro del grupo que se está estudiando; se identifica con él de tal manera que el grupo lo considera uno más de sus miembros" (p. 277). En la participación del investigador en el grupo es que se diferencia de 
la sola observación que se usa para la recogida de datos sobre comportamiento no verbal.

La observación participante conlleva la implicación del investigador en una serie de actividades durante el tiempo que dedica a observar a los sujetos objeto de observación en sus vidas diarias, y participar en sus actividades para facilitar una mejor comprensión. Para ello es necesario acceder a la comunidad, seleccionar las personas clave, participar en todas las actividades de la comunidad que sea posible, aclarando todas las observaciones que se vayan realizando mediante entrevistas (ya sean formales o informales), tomando notas de campo organizadas y estructuradas para facilitar luego la descripción e interpretación. Se reitera que es una investigación de aula y todo lo dicho se hace en el aula durante la jornada escolar.

Las razones por la cual se opta este instrumento son muy sencillas, primero, porque resulta útil en estudios exploratorios, descriptivos y orientados a la generación de interpretaciones teóricas $y$, segundo, porque otorga una mejor comprensión de lo que está ocurriendo en la cultura, y da credibilidad a las interpretaciones que hace de la observación.

Para realizarla, es necesario considerar una serie de actividades y procedimientos tales como establecer relaciones, seleccionar informes clave, los procesos para dirigir las observaciones (qué y cuándo observar), tomar notas de campo y describir los hallazgos. Además, es importante el asunto de la ética, ya que es necesario comunicar a la comunidad el objetivo de la observación.

\section{La entrevista en profundidad}

La entrevista es la técnica más empleada en las distintas áreas del conocimiento. En un sentido general, se entiende, siguiendo a Campoy y Gomes (2009) "como una interacción entre dos personas, planificada y que obedece a un objetivo, en el que el entrevistado da su opi- nión sobre un asunto y, el entrevistador, recoge e interpreta esa visión particular" (p. 288).

Cuando se habla de entrevista, con frecuencia se identifica en una técnica de investigación estructurada como las encuestas de actitud o de opinión y los cuestionarios. Sin embargo, cada vez más se va utilizando la entrevista en profundidad, también conocida como cualitativa, no estructurada, abierta o no estandarizada.

En la metodología cualitativa, la entrevista en profundidad se entiende como los encuentros reiterados cara a cara entre el investigador y el entrevistado, que tienen como finalidad conocer la opinión y la perspectiva que un sujeto tiene respecto de su vida, experiencias o situaciones vividas.

La entrevista en profundidad tiene mucho en común con la observación participante, pero se diferencia de esta en el escenario y situaciones en los que tiene lugar la investigación. Mientras que los observadores participantes realizan sus estudios en situaciones de campos naturales y los entrevistadores en situaciones específicamente preparadas. Además, el observador participante obtiene una experiencia directa del mundo social, mientras que el entrevistador obtiene esa visión mediante el relato del otro.

Este tipo de entrevista se caracteriza porque permite más comprender que explicar se basa en respuestas subjetivamente sinceras, cambia el sentido de la escucha, pues el entrevistador no evalúa las respuestas, sino que las oye con bastante interés. También permite la exploración de uno o dos temas en detalle y la información que se obtiene es contextualizada (personas, lugar, entre otros).

Ficha diagnóstica del estudiante

\section{Resultados}

En este sentido se ha realizado la interpretación y el análisis de los resultados de la investigación. De aquí saldrán los elementos para 
plantear las conclusiones. A este respecto Taylor y Bodgan (1986) afirman:

En las investigaciones cualitativas, el análisis de datos no es una fase del proceso de investigación sino un proceso en continuo progreso, dinámico y creativo, que se da en simultáneo con la recolección, la codificación y la interpretación y escritura narrativa de los datos (p. 158).

Una vez realizado este proceso se reorganizaron los datos por medio de códigos o categorías que sustentan el trabajo interpretativo con los datos. En los implementos presentados se ejecutó un trabajo de codificación como lo muestra la figura 2. Etiquetamiento.

Figura 2. Etiquetamiento

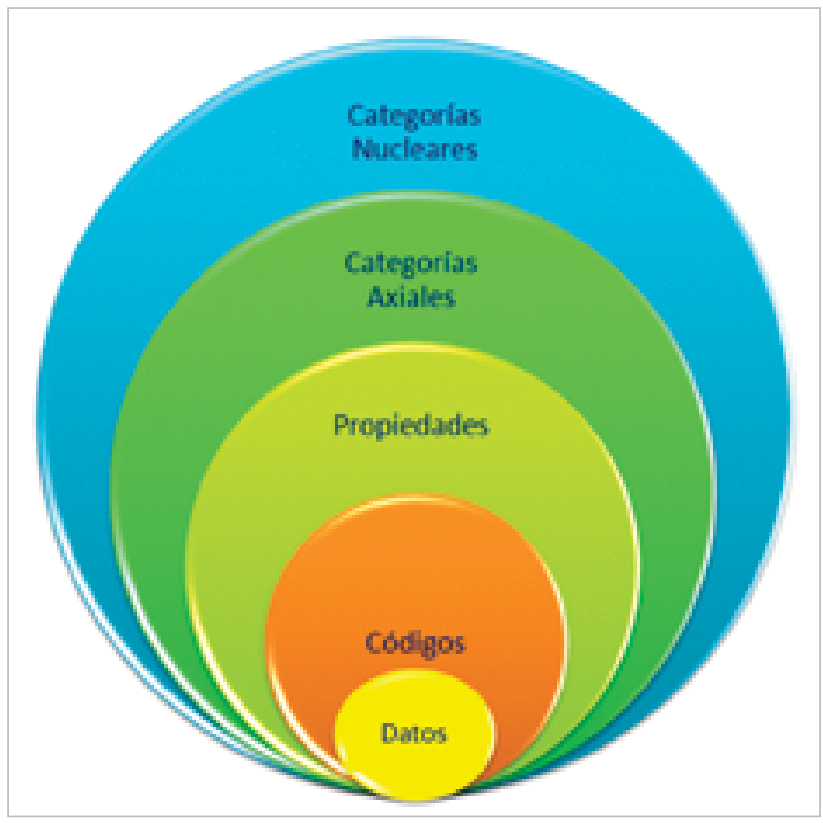

Al respecto Strauss y Corbin (2004) señalan:

El propósito de la codificación axial es comenzar el proceso de reagrupar los datos que se fracturaron durante la codificación abierta. En la codificación axial las categorías se relacionan con sus subcategorías para formar unas explicaciones más precisas y completas sobre los fenómenos (p. 135).

A continuación, se relacionaron las categorías nucleares que integraron el presente es- tudio y sobre las cuales se realizó el análisis de los datos: Concepciones, imaginarios y buenas prácticas de la enseñanza y el aprendizaje a partir de la danza en un contexto escolar; La danza como un proceso de formación interdisciplinar que involucra el aprendizaje con la expresión de emociones en niños de 5 a 7 años, y Momentos pedagógicos para emplear la danza como estrategia interdisciplinar de aprendizaje.

Categoría Nuclear 1. Concepciones, imaginarios y buenas prácticas de la enseñanza y el aprendizaje a partir de la danza en un contexto escolar.

Las categorías axiales que obtuvieron mayor frecuencia y relación con el primer objetivo del estudio de investigación: Diagnosticar las concepciones y prácticas que se tienen en la escuela con relación a la danza como estrategia interdisciplinar de enseñanza-aprendizaje, fueron las siguientes:

- Manifestaciones del aprendizaje situado a partir de la expresión artística.

- La didáctica de la danza como estrategia para superar los retos de los estudiantes.

- El fortalecimiento y la divulgación del proceso de la danza como estrategia de aprendizaje en la escuela.

A partir de estos elementos se hace el siguiente análisis:

Es claro que la danza en su manifestación y al ser trasmitida por el cuerpo, recreó experiencias y vivencias del mundo de la vida, que se convirtieron en posibilidades de aprendizaje y enseñanza. Bailar no solamente involucró el cuerpo sino también la mente, el espíritu; promovió momentos de alegría, compañerismo y convivencia.

Cuando se implementó para la enseñanza desde el descubrimiento, logra que el estudiante, a partir de la motivación, imitación, ejercicios corpóreos y consecutivos descubra y apro- 
pie los conceptos que se pretenden en el acto de enseñar, ya que involucra el pensamiento, el razonamiento y posibilita el desarrollo de las inteligencias ligadas con la participación, la expresividad de lo que se siente y afina la observación; elementos importantes para el acto de aprender (P.E.3). (Padre de familia entrevistado No. 3): "Me gusta mucho cuando aprende desde el arte - Me es gratificante verla cantar, bailar y hacer cosas que antes no hacía" (abril, 2017).

Con la mediación del docente, el estudiante resolvió sus interrogantes. De ahí la necesidad de la realización de las buenas prácticas para la enseñanza y el aprendizaje. Fue importante en el proceso de investigación el uso del trabajo grupal y el acompañamiento permanente del docente para proponer alternativas pedagógicas a las diversas dificultades que se presentaron en el aula. Esto apoya el proceso de cooperativismo, compañerismo y seres competentes.

De esta manera, el aprendizaje al ser interiorizado y manifestado en lo que aprendió, el estudiante mostró su potencial y su habilidad, lo que permitió evaluar el desempeño en el saber hacer, base del trabajo por competencias (P.E.1). (Padre de familia entrevistado No. 1): "Aprende actividades artísticas - La profesora genera gusto en el aprendizaje" (abril, 2017). Así también lo expresa Durán (1995) cuando menciona:

La danza enseña al niño tanto la conciencia del cuerpo como el control de sus movimientos. Este aprendizaje le sirve no solo para los juegos, el deporte y la vida diaria, sino para ampliar su percepción sensible; sobre todo, aprende a relacionar el lenguaje del cuerpo con el lenguaje verbal (p. 109).

En las familias de los niños se fomentaron espacios de conversación, los niños expresaron y manifestaron lo sucedido en la clase haciendo que en las familias se generaran espacios de exposición, confrontación y motivación como lo manifiesta (P.E.6) (Padre de familia entrevistado No. 6). "Se estimulan para que actúen artísticamente y no se apenan como sucedía al principio, Ahora los veo más activos y espontáneos, Quieren actuar cuando hay oportunidad" (abril, 2017).

El apoyo de los padres de familia y la motivación del docente buscó crear alternativas de aprendizaje desde la danza, haciendo que se vinculen áreas del conocimiento impartidas en el currículo escolar. La didáctica en las áreas del conocimiento con la estrategia presentada en este estudio, unió nuevas posibilidades de aprendizaje significativo y de enseñanza. Como lo manifiesta (P.E.3) (Padre de familia entrevistado No. 3): "No solo se aprende con el lápiz y el cuaderno, se aprende muchas cosas con la danza, Les gusta" (abril, 2017).

En este sentido, se encontraron aportes de teóricos como Fuentes (2008) quien afirma:

La danza es un vehículo pedagógico de gran importancia porque no sólo enseña pasos y movimientos o ayuda a la expresión artística y emocional de los niños, sino que además transmite y fomenta valores éticos como la tolerancia, la cultura del esfuerzo, el trabajo en equipo, la resolución de problemas y la independencia. Además, sirve para conocer y comprender otras culturas y tradiciones a través de piezas coreográficas (argumentos e historias) y estilos de danza de diversos orígenes (p. 11).

Las prácticas manifestadas en el aula de clase, permitieron mostrar la danza como eje didáctico al realizar la enseñanza de contenidos y el logro de desempeños en las áreas básicas con claridad. Esta estrategia integradora ha permitido que el modelo de escuela nueva, que se orientó en la formación de la sede de los Cacaos, posibilitó que los niños de los diferentes grados y de diferentes edades, compartieran el mismo contexto y desarrollaran juntos en el momento de iniciar la adquisición de los elementos que se propusieron desde la danza, la historia, el paisaje, para que después de acuerdo a su grado y edades desarrollaran los conceptos y los manifestaran a través de un baile que se propone desde todos, mostrando 
claramente los desempeños y las dificultades individuales.

La didáctica de la danza se convirtió entonces en alternativa de enseñanza que integra el conocimiento y la apropiación de conceptos, el desenvolvimiento espontáneo y la creación (aspectos claves que indican que el desarrollo es integral). Así lo manifestaron en (P.E.4) (Padre de familia entrevistado No. 4): "Gusto por la didáctica de la profesora, la didáctica ha ayudado a superar los retos a mi hija" (abril, 2017). (P.E.7) (Padre de familia entrevistado No. 7) "Los niños están despiertos y atentos, han mejorado su participación en la clase" (abril, 2017). (P.E.1). (Padre de familia entrevistado No. 1):"Las clases se hacen más didácticas y alegres" (abril, 2017) el (P.E.4) (Padre de familia entrevistado No. 4) "Me ha demostrado que con la danza se puede aprender más sus actividades" (abril, 2017) y el (P.E.5) (Padre de familia entrevistado No. 5): "La forma de las clases son más dinámicas, aprenden más tiene conocimiento de las culturas del país" (abril, 2017).

Categoría nuclear No. 2. La danza como un proceso de formación didáctica que involucra el aprendizaje con la expresión de emociones en niños de 5 a 7 años.

Las categorías axiales que obtuvieron mayor frecuencia y relación con el segundo objetivo del estudio de investigación: "Relacionar la danza como proceso de formación didáctico con la proyección de la dimensión psicosocial", fueron las siguientes:

- Sentimientos de los estudiantes en sus vivencias escolares.

- La danza como un espacio para la espontaneidad y juego de roles.

- El acompañamiento familiar en el desarrollo de las actividades del niño en la escuela.

Las experiencias vividas en la etapa escolar dejaron huella en la estructuración de la personalidad de los seres humanos. El contar con ambientes favorables que permitieron su desenvolvimiento con los demás, en este caso con los compañeros de varias edades y grados, posibilitó valores y actuaciones implícitas en la convivencia escolar, tales como el liderazgo, apoyo, cooperación y trabajo en equipo.

Por otro lado, el sentir agrado al cantar, al moverse al danzar generó apropiación del aprendizaje y permitió el desarrollo cognitivo, emocional y corpóreo. Para esta experiencia se hizo el ejercicio de trabajar con los niños las técnicas del dibujo y el color, para interpretar, desde las teorías que brindan elementos de análisis, los sentimientos vividos en las experiencias de aula; también se le fue preguntando por el sentimiento expresado en el dibujo. Los colores y los sentimientos son asociaciones de experiencias universales que arraigan desde la infancia. La creatividad influye en este aspecto considerablemente.

Ese sentimiento es relacionado por un color, que muestra en algunos: alegrías, lealtad, amor y bondad, entre otros. En la siguiente gráfica se mostró el resultado de este trabajo tal como aparece en la F.E. (Ficha diagnóstica del estudiante).

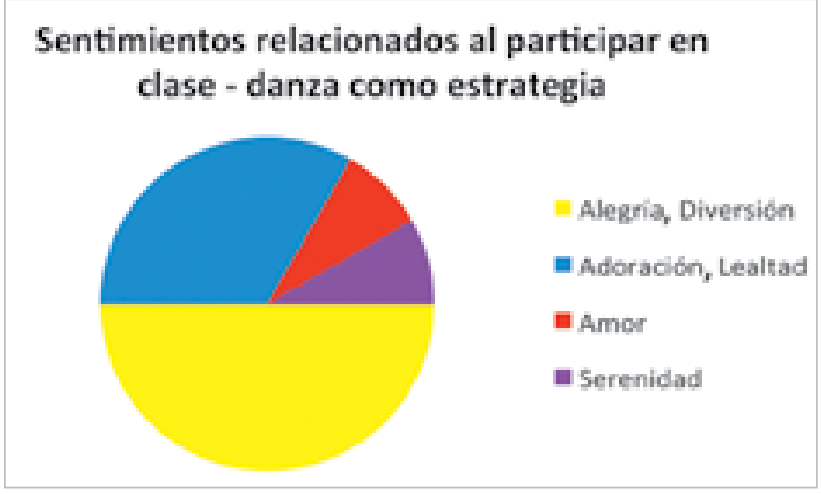

De un total de doce estudiantes que realizaron el dibujo, seis de ellos manifestaron alegría y diversión, expresando agrado, querer estar en la escuela y participar de las actividades propuestas, (F.E.2) (Ficha diagnóstica del estu- 
diante No. 2) “Dibujó nubes, soles, montañas, empleando varios colores, flores, dibujó a la profesora y se dibujó con los brazos extendidos. Se indaga por el movimiento que ejecuta el dibujo y manifestó: "Estoy bailando con mi profesora" (junio, 2017). Para los estudiantes que tienen la oportunidad de vincular la danza como estrategia en el proceso de aprendizaje expresaron tener un sentimiento de alegría concebido como: Diversión, agrado por estar en la escuela asistiendo y participando en ella, la inquietud manifestada en cada uno de los estudiantes en el desarrollo de la clase, qué ocurría, qué trajes usarían, qué danza bailarían.

El sentimiento de la alegría manifestado en la (F.E.6). (Ficha diagnóstica del estudiante No. 6) del grado primero permitió ver "un dibujo muy colorido, con pasto de colores, un arco iris, nubes, corazones; dibujó a la profesora danzando con vestuario de círculos y ella al lado imitándola, dibujó caritas felices en toda la hoja" (junio, 2017). Mostró claramente la emoción y la admiración plasmadas en el dibujo, expuso como se sintió al participar de las actividades, evidenció la alegría contagiosa que el docente transmitió a sus estudiantes, mostrando que la implementación de la danza como estrategia didáctica aportó la participación, la atención y la alegría. Expresó el estudiante, dibujando el arco iris, un momento de felicidad y empleó colores cálidos, vivos, fuertes y las figuras de corazones en el dibujo.

En este sentido los teóricos que se han acercado a estudiar el desarrollo socioemocional, desde la danza, permitieron afirmar la validez de lo planteado, según Lincovil (2016) “La danza permite que los niños y niñas pueden expresar los sentimientos de forma mucho más clara, sobre todo en aquellos que todavía no hablan, o aquellos niños a los que les cuesta expresarse verbalmente" (p. 1).

La danza, aparte de permitir la expresión de sentimientos, influye también enormemente en la relación de los niños y sus formas de comunicarse y desenvolverse como persona, elementos importantes para la educación las emociones y que están implícitos en el momento del aprendizaje. Quizá por esta razón no se evidenciaron cuando se trabaja en el aula con didácticas que desarrollan solo la competencia del saber, dejando de lado la competencia del ser (P.E.5) (Padre de familia entrevistado No. 5) "Niños más dinámicos y entusiasmados" (abril, 2017).

La familia como primera escuela en formación muestra más allá de los cuidados, precauciones y responsabilidades, debe aportar un apoyo incondicional y constante en el proceso educativo. La vinculación de los padres de familia en la educación de los hijos beneficia su autoestima, potencia el trabajo en equipo y permite la capacidad de formar seres exitosos.

Esta tarea conjunta permitió que los padres vean la necesidad de dejar que sus hijos asistan a la escuela P.E.1 y P.E.2. (Padres de familia entrevistados No. 1 y No. 2) "Apoyo del hogar. Le decimos que debe prestar atención. Mucho diálogo" (abril, 2017) y le brinda al educando una mejor comprensión para asimilar su proceso de enseñanza, tarea que le compete acompañar a los docentes, así se evidenció en la F.O.2. (Ficha de observación No. 2) cuya actividad integra a los padres de familia, estudiantes y comunidad en general de la escuela Los Cacaos, al presentarse la labor del campesino perteneciente a la región andina. "Comentaron sus experiencias de vida, alimentos preferidos, costumbres, y entre ellas destacamos la danza representativa de la vereda que es la Carranga" (abril, 2017).

Los padres de familia, al personificar con espontaneidad, asumieron su rol motivando a sus hijos para participar en la danza. "Los niños disfrutaron del momento, puesto que no habían visto a sus papás vestidos con el traje" (abril, 2017), aparte de ello demostraron interés y respaldo por la actividad. En el momento de la representación, los padres de familia portaron su traje como bailadores de vereda, 
relacionaron sus momentos de vida cotidiano manifestadas en la danza, las historias que cantan los grupos de vereda cuentan, narran y engalanan las celebraciones por los productos recibidos, las fiestas populares dadas en bautizos, matrimonios, entre otros. La unión y la participación lograron despertar espacios de afectividad, aprendizaje desde la danza, y se relacionaron con la región Andina.

Categoría nuclear No. 3 Momentos pedagógicos para emplear la danza como estrategia interdisciplinar en el aprendizaje.

Las categorías axiales que obtuvieron mayor frecuencia y relación con el tercer objetivo del estudio de investigación: Determinar los espacios y momentos pedagógicos didácticos a partir de la danza, son:

- La danza como estrategia interdisciplinar en las áreas de conocimiento.

- Atributos de un docente de artística transferidos a docentes de otras áreas.

- El fortalecimiento de la danza en el desarrollo de las diversas inteligencias de los estudiantes.

La danza como estrategia interdisciplinar para la enseñanza y el aprendizaje involucró la pedagogía del arte, del sentir, de la manifestación de emociones al aprender, y las relaciones con el entorno escolar. Por esta razón, se hizo uso de unos referentes conceptuales, descubrieron las metodologías artísticas capaces de trabajar en el ámbito de la didáctica, la creatividad y su aplicación en la educación.

En la F.O.6. (Ficha de observación No. 6) se estableció como objetivo "Identificar elementos didácticos relacionados entre la danza y las ciencias naturales, ciencias sociales, ética y valores, lenguaje" (marzo, 2017). Para la aproximación de la consecución de este objetivo en el trabajo realizado se tuvo como eje integrador la región amazónica y por lo tanto, tuvo como punto de partida del proceso de aprendizaje una danza llamada "boitata", que consiste en la representación de la labor de una boa que busca proteger la flora y la fauna de la región.

Allí se vio claramente cómo los niños y niñas caracterizaron "animales salvajes, vivenciaron los cuidados de la naturaleza y la conservación de la flora y la fauna" (marzo, 2017). También se dieron la oportunidad de descubrir los aspectos que componen la región amazónica.

Se reitera que la danza utilizada hace referencia a una leyenda, desde allí se hizo la conceptualización e interpretación de los componentes de la leyenda, partiendo de la aplicación de la danza teatro.

Se evidenció el interés, la atención y la habilidad para aprender por medio de la ejecución de la danza: "Sabe lo que está bailando, porque lo conoció imitando los movimientos explorando su cuerpo para recrear otros gestos como formas comunicativas" (marzo, 2017), "Ios niños realizaron los accesorios característicos de las tribus indígenas, en este caso trenzaron cordones y elaboraron collares representativos de los Ticuna colombiano - brasilero" (marzo, 2017).

Los niños lograron ser competentes, porque aprendieron, identificaron lo propuesto en clase y realizaron un trabajo cooperativo para manifestarlo en la danza. Se permitió a los niños que con la danza lograran distinguir la región amazónica en sus expresiones, formas de convivencia, laboreo, vestuario, y maquillaje.

Este proceso lo posibilita un docente que crea en las nuevas alternativas didácticas, que sea formado en arte o que le apasione, así es concebido por los directivos de la institución.

Entrevista a la Coordinadora: "Debe ser un ser sensible, humano y artista - con capacidad para realizar prácticas de aula innovadoras con capacidad de privilegiar la duda - con capacidad de transmitir las herramientas que les permitan a los estudiantes poner en práctica las 
operaciones del arte - Que tenga la capacidad de involucrar el juego como estrategia artística para el desarrollo de la creatividad, la competencia intelectual y la fortaleza emocional" ... Contar con una especialista en educación artística, que desde la danza ha fortalecido el razonamiento condicionado, el ordenamiento mental y el pensamiento creativo. Se ha conseguido el fomento de la curiosidad por el conocimiento, profundizado la perseverancia, desarrollado la disciplina, aumentando la capacidad de atención e incrementado la retención escolar. Se ha visto un cambio en la autoestima de los estudiantes, ampliación de la conciencia de la propia identidad y el desarrollo de destrezas de autocontrol. La promoción de un ambiente de colaboración entre padres de familia y estudiantes generando mayor compromiso hacia el proceso de sus hijos" (abril, 2017).

Hablar de la danza como estrategia integradora del currículo, no es solo hacer un enlace con los contenidos temáticos, tampoco es hacer actividades diversas y presentarlas en una danza. Va más allá, es partir del mundo de la vida y desde allí entrar al mundo del conocimiento y el desarrollo de los procesos mentales, para potenciar las competencias que lleven a desempeños desde el saber, saber hacer y ser.

Es integrar la evaluación en la manera como se desarrollan las inteligencias, las habilidades, las actuaciones y la claridad conceptual, en el hacer permanente de la clase, es posibilitar la inclusión, la aceptación del otro y la vinculación de la familia para que sea partícipe de este proceso y acompañe al estudiante en el seguimiento a los procesos ganados en la escuela.

\section{Conclusiones}

Cuando en la escuela se observa un grupo de estudiantes portando un traje que identifica una danza, se determina por lo general que: Los niños se están midiendo el vestuario en un ensayo, o van a una representación de una danza para un evento cultural importante. Considero que va más allá. La puesta en escena, el montaje de una danza en un contexto escolar genera un espacio de armonía integrando a los niños en edades variadas, en conocer diferentes manifestaciones integrando la educación y las artes, transmitirlas e implementarlas desde las áreas de conocimiento, en el impacto y atención que genera al vivenciar una danza, una tradición de un pueblo, en la manifestación expresiva que genera agrado y enseña a que los niños participen y conozcan desde la escuela.

En consecuencia, al integrar la danza como estrategia para la enseñanza que se planteó en este proyecto de estudio, es el reconocer y divulgar el aprendizaje permanente de nuevas alternativas para la enseñanza. Como primera medida se realizó un diagnóstico de los momentos pedagógicos, antecedentes que evidenciaron el sistema educativo que se estaba implementando, una vez socializada la propuesta con la comunidad educativa; se relacionó la danza como estrategia didáctica promoviendo espacios y ambientes escolares diferentes, estos se determinaron en momentos pedagógicos que apoyaron la efectividad de la estrategia aplicada.

Teniendo en cuenta el propósito de estudio que se relacionó con el objetivo general y la pregunta problema que menciona la danza como una estrategia didáctica que proyecta la enseñanza y el aprendizaje de los niños y niñas de 5 a 7 años del colegio Agroecológico Holanda, sede Los Cacaos. Para lograrlo, la estrategia se desarrolló en situaciones cotidianas en el aula de clase y siempre con el uso de orientaciones pedagógicas que involucraban el aprendizaje cognitivo, lingüístico, integral, corporal; relacionándolo con la danza.

Se evidenciaron momentos en que los niños atendieron, participaron y descubrieron potencialidades y habilidades que con esfuerzo y dedicación están superando. Están fortaleciendo su expresividad en el aula, en sus hogares, en compañía y con apoyo de los padres de familia 


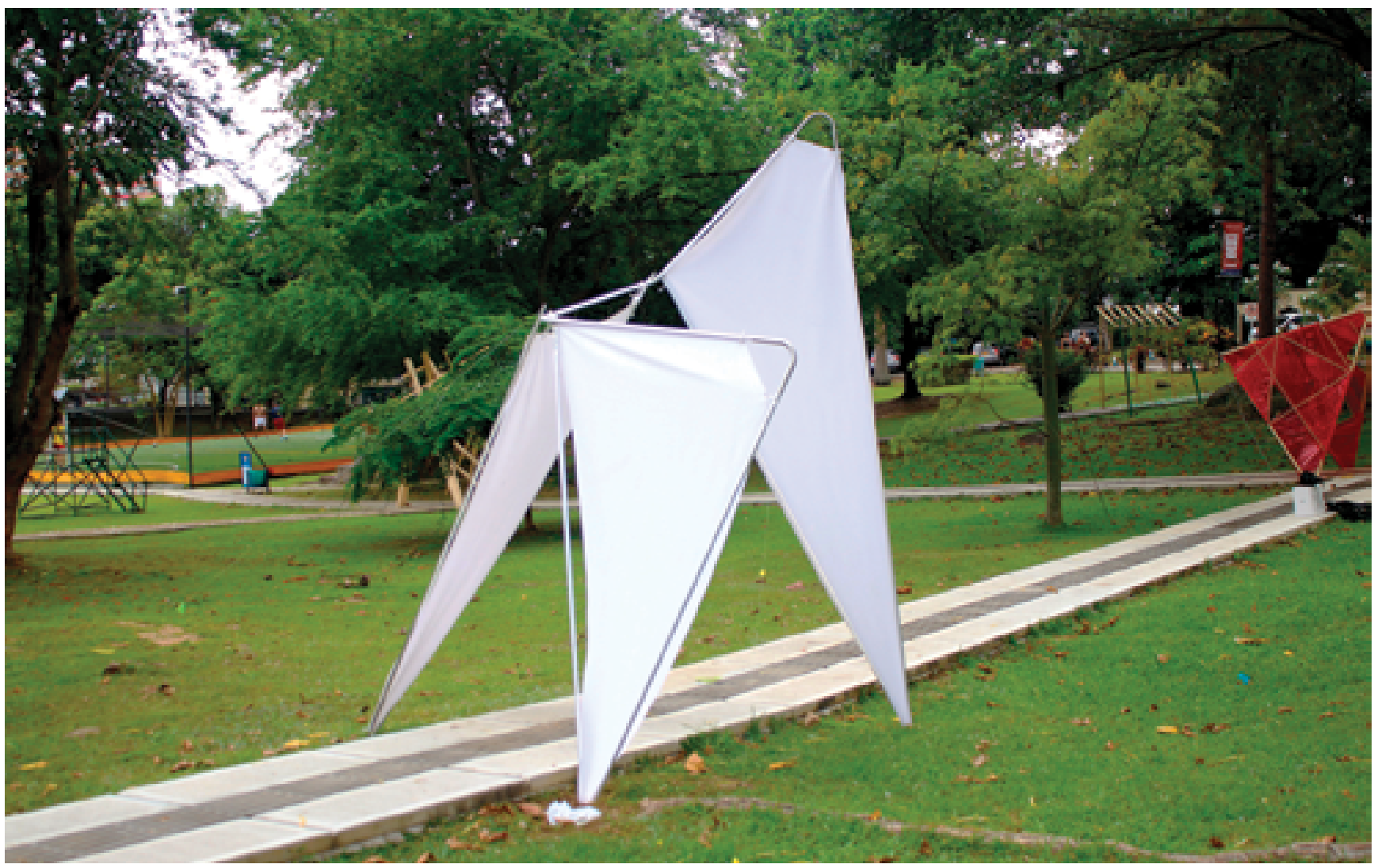

Proyecto Proyecto Ki.

que también son protagonistas de este trabajo de investigación. Los niños al contar y socializar las experiencias vividas, están dando a conocer aquello que les llamó la atención y por tanto logró ser más competente. Todo esto va acompañado de la manifestación del cuerpo a través de la danza, la comunicación y la relación de su yo con el entorno.

Al finalizar -aunque en realidad es un iniciar debido a que la propuesta es una nueva puerta hacia el aprendizaje- la aplicación de la estrategia se encontró que algunos resultados se alinearon o dieron respuesta al objetivo general, determinando la efectividad de la danza como estrategia didáctica en los niños y niñas de 5 a 7 años de la escuela Los Cacaos que integraron las clases de la muestra tomada y evidenciada en la escuela durante el período de los años 2016 y 2017 . Es necesario enfatizar que el contexto rural en el que se desenvuelven las familias acarrea un proceso de asimilación, sensibili- zación y oportunidad de brindar una estructura cambiante a la que ellos vivenciaron en su momento académico, o por el contrario, a la metodología de enseñanza que nunca conocieron.

Es meritorio el trabajo desarrollado con la implementación de la estrategia, los niños disfrutan del proceso, son felices, verlos llegar a la escuela y estar atentos a las actividades por realizar, el hecho de relacionar las áreas de conocimiento con la danza hace que los estudiantes conocieran y asociaran presaberes con el movimiento, la expresión y la manifestación de agrado al vivenciarlas. También la participación frente a sus compañeros, la toma de decisión para opinar, dar criterio, asumir miedos, expresar emociones y sentimientos en la ejecución de las actividades resaltando el arte educativo.

Los niños incrementaron su potencial de asociación, al escuchar la danza contando en qué actividad participaron y qué aprendieron de ella, manifestaron agrado al oír y trabajar 
en sus actividades durante la jornada escolar, estableciendo momentos sorpresivos, pues en ciertos espacios de la clase al oír la danza, se levantan del puesto y bailan. Seguidamente al terminar seguían desarrollando la actividad, lo cual este instante permitió establecer espacios de pausas activas, beneficiando a los niños para continuar con el proceso empleado desde la estrategia.

Motivó potenciar sus competencias, los niños al participar en las clases empleando la estrategia fueron desarrollando su pensamiento crítico, resolución de problemas, buscando alternativas para aprender y ayudar al compañero que no lograba de primer impulso comprender presaberes, se han convertido en niños padrinos, se designó así al nivel de comprensión que unos desarrollaban más rápido que otros, pero su finalidad era que todos participaran de la actividad estructurada. Asumen puestas en escena frente al público, se potenció bastante la expresividad y el vencer la pena, sobre todo en los niños que han estado los dos años consecutivos en la implementación de la estrategia.

La aplicación de los instrumentos, al diagnosticar las prácticas en la escuela con relación a la danza como estrategia de aprendizaje, arroja como resultado aspectos positivos que involucraron a los niños a expresarse, se comunicaron y manifestaron sus gustos, ideas y opiniones. Se motivó a la expresión por medio del canto interpretado en la danza, de la imitación de movimientos potenciando la motricidad fina y gruesa al desplazarse, al coser, al tejer, entre otros; se comunica de manera verbal y no verbal descubriendo que no solo con el habla transmite un mensaje, también lo puede manifestar con el cuerpo.

Se saca como conclusión que la estrategia permite que, empleando la danza con las otras disciplinas del saber, interioricen conocimientos y complementen su currículo escolar. No solo se aprende con el lápiz y con el cuaderno, se aprende con la danza y les gusta. Manifes- taron participación, actitud y disposición para atender a la didáctica empleada por él docente.

El impacto de la estrategia empleada potenció la expresividad, la participación y la competencia de la mayoría de los estudiantes del aula de clase, puesto que favoreció como primera instancia la adecuación de espacios lúdicos, generó ambientes que permitían que los niños se vincularan a la estrategia, relacionando la danza con las áreas de conocimiento, superar miedos y potenciar su autoestima al verse lo que explica, al interactuar con el otro y dar su opinión de lo que le llamó la atención en el momento de la participación de la clase.

Se potenciaron las habilidades y destrezas que los niños pueden tener desde el momento que participaron en actividades relacionadas con el arte, despertó atención y formas de manifestar sus presaberes, logrando una apropiación y asociaciones que le permiten en su momento desarrollar habilidades cognitivas y motrices, y así relacionarlas con las personas que lo rodean. Lograron un proceso de caracterización, asumieron un rol de captación y desenvolvimiento; lo cual contribuye y fortalece la aplicación de la estrategia en el lapso de dos años de su implementación.

Finalmente, la estrategia implementada apoyo el proceso del descubrimiento de habilidades cognitivas, sociales e integrarles, destrezas que con el tiempo y proceso debido de conservación generarán seres inteligentes que se motiven a conocer cada día más de dónde son y qué pueden hacer para ser personas felices aprendiendo desde la danza.

\section{Referencias}

Campoy, T., y Gomes, E. (2009). Técnicas e instrumentos cualitativos de recogida de datos. Material no publicado. Recuperado el 8 de diciembre de 2016 de http://www2. unifap.br/gtea/wp-content/uploads/2011/10/T_cnicase-instrumentos-cualitativos-de-recogida-de-datos1.pdf

Durán L. (1995). La importancia de la danza infantil en el proceso educativo. Universidad Veracruzana. México. Recuperado de http://cdigital.uv.mx/bitstream/123456789/4 164/1/199542P107.pdf 
Ezpeleta, J. (2004). Innovaciones educativas. Reflexiones sobre los contextos en su implementación. Revista Mexicana de Investigación Educativa, 9,21. Recuperado de www. oei.es/reformaseducativas/reflexiones_innovaciones_ educativas_ezpeleta.pdf

Fuentes, S. (2008). Pedagogía de la danza. Cuadernos de danza, 3. Bizkaia: Danza Getxo. Recuperado de http://www. libreriayorick.com/teatro/danza/2868-pedagogia-dela-danza-sylvia-fuentes.html

Flores, J., Gómez, G. R., \& Jiménez, E. G. (1999). Metodología de la investigación cualitativa. Málaga: Aljibe.

Heller, E. (2004). Psicología del color. Editorial Gustavo Gili. Barcelona. España. Recuperado de http://www.sncpharma.com/assets/lib/kcfinder/upload/files/libros\%20snc/ Psicologia\%20del\%20Color.pdf

Lincovil, M. J. (2016). Danza en la educación inicial: ¿Cuáles son los beneficios? Elige educar. Recuperado de http:// www.eligeeducar.cl/cuales-son-los-beneficios-de-incorporar-la-danza-la-educacion-inicial
Ogando, F. (2004). Reporte sobre estado de la investigación en gestión escolar, práctica pedagógica y calidad educativa. Recuperado de http://ww2.educarchile.cl/UserFiles/P0 001\%5CFile\%5CFERNANDOOGANDOInformedelnvesti gacion.pdf

Strauss, A., Corbin, J. (2002). Bases de la investigación cualitativa: técnicas y procedimientos para desarrollar la teoría fundamentada. Medellín, Colombia: Editorial Universidad de Antioquia. Recuperado de https://diversidadlocal.files.wordpress.com/2012/09/bases-investigacioncualitativa.pdf

Taylor, S., y Bogdan, R. (1987). Introducción a los métodos cualitativos de investigación. Paidós. España. Recuperado de http://mastor.cl/blog/wp-content/ uploads/2011/12/Introduccion-a-metodos-cualitativosde-investigaci\%C3\%B3n-Taylor-y-Bogdan.-344-pagspdf.pdf

Woods, P. (1987). La etnografía y el maestro. La etnografía en la investigación educativa. Paidós. Barcelona. Recuperado de https://es.scribd.com/document/326167869/ Woods-Peter-La-escuela-por-dentro-La-etnografia-enla-investigacion-educativa-pdf 